\title{
Current and potential future applications of human stem cell models in drug development
}

\author{
Mark Donowitz, ${ }^{1,2}$ Jerrold R. Turner, ${ }^{3}$ Alan S. Verkman, ${ }^{4}$ and Nicholas Constantine Zachos ${ }^{1}$ \\ 'Division of Gastroenterology, Department of Medicine, and 2Department of Physiology, Johns Hopkins University School of Medicine, Baltimore, Maryland, USA. ${ }^{3}$ Department of Pathology, Brigham and \\ Women's Hospital and Harvard Medical School, Boston, Massachusetts, USA. ${ }^{4}$ Departments of Medicine and Physiology, UCSF, San Francisco, California, USA.
}

$\mathbf{T}$ he use of model systems is of obvious importance for understanding human physiology and pathophysiology. However, the value of using human models for drug development has only relatively recently been appreciated. An FDA review of drug development reported that $80 \%$ to $90 \%$ of drug candidates entering phase I clinical trials fail, with the most common causes of failure being lack of efficacy and toxicity (1-3). Lack of efficacy accounted for $57 \%$ of failures. Toxicities, most commonly involving the cardiovascular system, liver, gastrointestinal system, and CNS, accounted for drug failure in $35 \%$ and $28 \%$ of phase I and phase II clinical trials, respectively (3). The ability to simply and reliably identify and exclude drugs that lack sufficient efficacy or cause serious toxicities has the potential to greatly increase the success rate of costly and time-consuming clinical trials. Current preclinical models are, however, insufficient to detect some toxicities. For example, one study reported that $57 \%$ of 221 human toxicity events caused by 150 compounds were not predicted by preclinical rodent studies (4). Thus, although transformed cell lines and animals are the primary models currently used, it seems imperative to develop better models that recapitulate human physiology and pathophysiology. Herein, recent advances in primary human culture models, their utility as preclinical testing platforms for drug target validation, and their importance in the future of drug discovery will be discussed.

\section{Species-specific differences affect drug candidate identification}

Intestinal and hepatic drug absorption and metabolism are dictated by factors including the chemical properties of the drug candidate (e.g., solubility, permeability), the physiology of the intestinal tract (e.g., transit time, blood flow), and patient phenotypes (e.g., age, sex, polymorphisms in drug-metabolizing enzymes, disease state). Many of these aspects of drug handling exhibit species specificity; common examples of differences between humans and animal models include regional abundance and specificity of drug-metabolizing enzymes $(5,6)$. For example, 34 cytochrome P450 gene families are involved in drug metabolism in mice, while only eight are major participants in humans (5). Such differences confound extrapolation of drug data from animal models to humans.

The "organ-on-a-chip" program is one potential approach to overcome species-specific obstacles and has been collaboratively funded by the National Center for Advancing Translational Sciences, Defense Advanced Research Projects Agency, and FDA. The program goal is to test whether preclinical studies using normal human tissue would reduce drug failure rates. Primary cultures from up to 11 human organs were generated, grown in bioengineered platforms, and scaled to represent the fraction of blood flow that each organ receives in humans so that drug metabolites from each organ could be evaluated for toxicity in downstream organs, as would occur in vivo (6). In parallel with these experimental studies, a working group of scientists from pharmaceutical companies (the Innovation and Quality Consortium's Microphysiological Systems working group) was invited to opine on the need for the new models and technology from a pharma perspective $(3,7)$.

Conflict of interest: ASV is a named inventor on multiple patents on small-molecule modulators of ion transport, whose rights are owned by the University of California.

Copyright: $\odot 2020$, American Society for Clinical Investigation.

Reference information: / Clin Invest. 2020;130(7):3342-3344. https://doi.org/10.1172/JCI138645.

\section{Advances with human stem cell-based models}

Three major technological advances have enabled the use of human stem cellderived cultures. The first advance was the molecular identification of actively dividing intestinal stem cells by the Clevers laboratory $(8,9)$. This pioneering discovery included methods to isolate and culture stem cells to generate a primary cell culture model made up of human intestinal epithelial cells (here referred to as human intestinal enteroids). Second, the Wells laboratory developed methods to differentiate inducible pluripotent stem cells (iPSCs) into a multicellular culture model of the fetal intestine that includes epithelial cells and an adjacent mesenchyme (referred to as human intestinal organoids). Both the stem cell and iPSC culture methods have been modified in subsequent studies to grow liver stem cells and organoids. These tools have enabled elucidation of new principles underlying the physiology of intestinal ion and nutrient transport (10) and provided new insights into the pathophysiology of host interactions with enteric pathogens, including viruses (rotavirus, norovirus, coxsackie virus, and SARS-CoV-2) (11), bacteria (multiple E. coli, Clostridium difficile, and Shigella spp.), and parasites (cryptosporidia, Toxoplasma gondii, helminths) $(12,13)$. The recognition that some disease-associated phenotypic changes are maintained in these culture models has also led to use of patient-derived enteroids for studies of noninfectious gastrointestinal diseases, including inflammatory bowel disease, celiac disease, radiation- and chemotherapy-induced injury, cystic fibrosis, and colon cancer. Patient-derived stem cell biobanks are rapidly expanding. The third major advance has been the development of platforms, including simple culture dishes, Transwell filters, and microphysiological systems, that facilitate the growth 
and differentiation of human stem cells. For example, microphysiological systems can include multicellular environments within biopolymer or tissue-derived matrices with distinct configurations and physical properties such as stiffness, incorporation of mechanical forces modeling the shear stress of luminal and vascular flow and the repetitive pulsations of cardiac and peristaltic contractions, and integration of multiple organ-on-a-chip devices to recapitulate the complexities of in vivo metabolism $(14,15)$. Further development of these devices will likely better model interactions between epithelial cells and other cell types, such as endothelium, smooth muscle, immune, and inflammatory cells; neural cells; and even nonhuman components, such as the microbiome.

As technology advances, experience to date offers some insight into the potential applications of human cells in drug development. For example, swelling assays performed in spheroidal small intestinal and rectal enteroids, as well as 3-dimensional cultures of nasal epithelium, have been useful in screening and evaluating correctors and potentiators of mutant cystic fibrosis transmembrane conductance regulator chloride channels that underlie cystic fibrosis (16). Responses to chemotherapy have been predicted using cancer-derived stem cell cultures, although the effectiveness of this approach over a prolonged period in the face of continued tumor stem cell mutation has not been established (17). A particularly persuasive example of human stem cell use in drug development comes from the Ingber group, who developed liver-on-a-chip models that included two or four human cell types (hepatocytes, endothelial cells, stellate cells, Kupffer cells) and compared drug metabolism in rat-, dog-, and human-derived models (18). The human model predicted multiple types of hepatotoxicity that occur in humans that were not predicted by the animal liver models or only exhibited hepatic toxicity at much higher concentrations than those that damaged human livers. These liver-on-chip models were able to predict hepatocyte toxicity, idiosyncratic hepatitis, cholestasis, lipid droplet formation in Kupffer cells, and stellate cell toxicity. In one example of the potential benefits of human liver-on-chip models, patients died in a phase I trial (19) as a result of liver toxicity that was not predicted by animal models. If the human toxicity had been known, it might have prevented the initiation of human studies. Conversely, other drugs with minimal or no hepatotoxic effects in human organoid studies were abandoned by pharma based on toxicity in animal studies (18). Human liver-on-chip systems might have allowed further drug development in these cases.

Given the progress in physiologic, pathophysiologic, and pharmacologic studies using human stem cell-derived systems, many pharmaceutical companies have now established internal programs or are collaborating with academic or commercial groups that are studying these model systems $(3,7,14)$. Though the details of these programs have not been publicly disclosed, based on preliminary publications and privileged discussions $(3,7,14)$, it appears that human cell-based in vitro systems are being used for early toxicity testing of promising drugs, pharmacokinetic analyses, and modeling of pathways that are dysregulated in complex human disorders, such as inflammatory bowel disease and host-pathogen interactions. It is envisioned that these model systems could also be used for target identification, high-throughput screening, lead optimization and selection, and preclinical pharmacokinetic and pharmacodynamic testing.

\section{Future directions for human stem cell-derived models}

Despite these advances and potential applications, it remains to be seen whether human stem cell-derived cultures will prove useful in reducing drug failures due to limited efficacy or toxicity, significantly decrease the use of animals in drug development, or accelerate speed and/ or reduce costs of drug development. It is also unclear whether human stem cell cultures will be used alongside or instead of animal and cell line models. Challenges include the relatively recent introduction of stem cell cultures as drug development tools and the need for continued maturation of stem cell-derived models to more closely resemble intact organs, especially using coculture of nonepithelial intestinal cells and inclusion of the microbiome (20, 21). It is advised that FDA rules be amended to include roles for human stem cells. For example, the FDA requires testing in two animal models before proceeding to human clinical trials. These rely on adsorption, distribution, metabolism, excretion, and toxicology (ADMET) evaluation in appropriate models, which is interpreted as only referring to intact animal models. At this time, stem cell-derived models are just another ex vivo preclinical model that has not been rigorously shown to reflect intact humans any more than other models used for drug development. In fact, other advances have occurred at the same time as development of stem cell methods that need to be evaluated for their role in drug development. Such advances include humanized animals, computational models including humanized computational models, and multiomic approaches (22). Ideally, characterization of the large data sets that can be compared with omics data from intact humans will enhance understanding of similarities and differences between animal models, intact humans, and stem cell cultures, including human cultures, and lead to improved predictions of patient responses.

What are the likely future applications of human stem cell models in drug development? This question will not be answered until results of studies using human stem cell models are compared with patient outcomes. Will the use of these human preclinical models increase success rates and reduce occurrence of unexpected toxicities in human trials? What criteria will the FDA define as necessary and sufficient for use of stem cells in the drug approval process? Although the FDA has established internal programs for studying stem cell models, indicating its belief in the potential importance of these models, data using any human stem cell models have yet to be submitted to the FDA for consideration as part of a drug application $(3,14)$. In the meantime, stem cell culture models continue to increase in complexity and more closely approximate the organs they represent, disease models using human stem cells have been shown to duplicate clinical aspects of the diseases and provide new insights concerning pathophysiology, new potential drug targets continue to be identified, and human stem cell-based biobanks are growing, allowing consideration of biodiversity. It has been predicted that increased use of stem cell models will provide a twofold 
reduction in time for drug development and a fivefold reduction in cost (14). This will only be realized, and certainly accelerated, if results of studies using stem cell models are documented alongside similarities and differences with studies of the same conditions in human patients.

\section{Acknowledgments}

This article was supported in part by $\mathrm{NIH} /$ National Institute of Diabetes and Digestive and Kidney Diseases R24 P30 DK72517, "Translational Approaches to Develop Drug Therapy for Diarrhea.”

Address correspondence to: Mark Donowitz, Ross 925, Johns Hopkins University School of Medicine, 720 Rutland Avenue, Baltimore, Maryland 21205, USA. Phone: 410.955.9675; Email:mdonowit@jhmi.edu.

1. Kola I, Landis J. Can the pharmaceutical industry reduce attrition rates? Nat Rev Drug Discov. 2004;3(8):711-715.

2. Seruga B, Ocana A, Amir E, Tannock IF. Failures in phase III: causes and consequences. Clin Cancer Res. 2015;21(20):4552-4560.

3. Fabre K, et al. Introduction to a manuscript series on the characterization and use of microphysiological systems (MPS) in pharmaceutical safety and ADME applications. Lab Chip.
2020;20(6):1049-1057.

4. Olson $\mathrm{H}$, et al. Concordance of the toxicity of pharmaceuticals in humans and in animals. Regul Toxicol Pharmacol. 2000;32(1):56-67.

5 . Kasendra M, et al. Duodenum Intestine-Chip for preclinical drug assessment in a human relevant model. Elife. 2020;9:e50135.

6. Huh D, Hamilton GA, Ingber DE. From 3D cell culture to organs-on-chips. Trends Cell Biol. 2011;21(12):745-754.

7. Ewart L, et al. Navigating tissue chips from development to dissemination: a pharmaceutical industry perspective. Exp Biol Med (Maywood). 2017;242(16):1579-1585.

8. Barker $\mathrm{N}$, et al. Identification of stem cells in small intestine and colon by marker gene Lgr5. Nature. 2007;449(7165):1003-1007.

9. Sato T, et al. Single Lgr5 stem cells build crypt-villus structures in vitro without a mesenchymal niche. Nature. 2009;459(7244):262-265.

10. Yin J, et al. Molecular basis and differentiation-associated alterations of anion secretion in human duodenal enteroid monolayers. Cell Mol Gastroenterol Hepatol. 2018;5(4):591-609.

11. Lamers MM, et al. SARS-CoV-2 productively infects human gut enterocytes (published online May 1, 2020). Science. https://doi. org/10.1126/science.abc1669.

12. Dutton JS, Hinman SS, Kim R, Wang Y, Allbritton NL. Primary cell-derived intestinal models: recapitulating physiology. Trends Biotechnol. 2019;37(7):744-760.

13. Hill DR, Spence JR. Gastrointestinal organoids: understanding the molecular basis of the host-microbe interface. Cell Mol Gastroenterol
Hepatol. 2017;3(2):138-149.

14. Marx U, et al. Biology-inspired microphysiological systems to advance patient benefit and animal welfare in drug development [published online February 28, 2020]. ALTEX. https://doi. org/10.14573/altex.2001241.

15. Vernetti L, et al. Corrigendum: Functional coupling of human microphysiology systems: intestine, liver, kidney proximal tubule, blood-brain barrier and skeletal muscle. Sci Rep. 2017;7:44517.

16. Berkers $G$, et al. Rectal organoids enable personalized treatment of cystic fibrosis. Cell Rep. 2019;26(7):1701-1708.e3.

17. Sasaki N, Clevers H. Studying cellular heterogeneity and drug sensitivity in colorectal cancer using organoid technology. Curr Opin Genet Dev. 2018;52:117-122.

18. Jang KJ, et al. Reproducing human and crossspecies drug toxicities using a Liver-Chip. Sci Transl Med. 2019;11(517):eaax5516.

19. Institute of Medicine (US) Committee to Review the Fialuridine (FIAU/FIAC) Clinical Trials, Manning FJ, Swartz MN, eds. Review of the Fialuridine (FIAU) Clinical Trials. Washington, DC, USA: National Academy Press; 1995.

20. Noel G, et al. A primary human macrophageenteroid co-culture model to investigate mucosal gut physiology and host-pathogen interactions. Sci Rep. 2017;7:45270.

21. Jalili-Firoozinezhad S, et al. A complex human gut microbiome cultured in an anaerobic intestineon-a-chip. Nat Biomed Eng. 2019;3(7):520-531.

22. Brubaker DK, Lauffenburger DA. Translating preclinical models to humans. Science. 2020;367(6479):742-743. 